\title{
Investigation of a weak magnetic field effect on the in vitro catalytic activity of adenosine deaminase and xanthine oxidase
}

\author{
Kadir Batcioglu ${ }^{1}$, Metin Dogan ${ }^{2}$, Ayse B. Uyumlu ${ }^{1}$, Basri Satilmis ${ }^{1}$, Nevzat Bayri ${ }^{3}$, \\ Selcuk Atalay ${ }^{3}$ and Hakan Demirtas ${ }^{4}$ \\ ${ }^{1}$ Department of Biochemistry, Faculty of Pharmacy, University of Inonu, Malatya, Turkey \\ ${ }^{2}$ Department of Radiology, Faculty of Medicine, University of Inonu, Malatya, Turkey \\ ${ }^{3}$ Department of Physics, Faculty of Art and Sciences, University of Inonu, Malatya, Turkey \\ ${ }^{4}$ Department of Epidemiology and Biostatistics, University of Illinois at Chicago, Chicago, USA
}

\begin{abstract}
The effect of a weak magnetic field (MF) on adenosine deaminase (ADA) and xanthine oxidase (XOD) activities have been investigated. A $50 \mathrm{~Hz}$ uniform MF was generated, and the magnitude of the field was kept constant at $5.8 \mathrm{mT}$. The changes in ADA activity over time were significantly different in and out of the MF; MF caused a steeper decline in ADA activity compared to the situation when no MF is present. In addition, MF caused a significant increase in XOD activity. There were no significant time-related changes for either enzyme in the absence of the MF. We suggest that a weak MF affects enzymatic systems.
\end{abstract}

Key words: Magnetic field — Adenosine deaminase - Xanthine oxidase

Xanthine oxidase (XOD; EC 1.2.3.2) catalyses the oxidation of many substrates including purines (e.g. xanthine and hypoxanthine), pteridines, heterocyclic molecules, and aldehydes (Lacy et al. 1998).

The reaction of xanthine with XOD produces both superoxide and hydrogen peroxide directly (Takanami and Nakayama 2011). In addition, XOD contains transition metals required for catalysis, namely molybdenum and iron which play a key role during the catalytic activity of XOD. The kinetic studies indicate a path for electron transfer as follows: Xanthine $\rightarrow \mathrm{Mo} \rightarrow$ Flavin $\rightarrow$ Fe-S cluster (Doyle et al. 1996).

Adenosine deaminase (ADA; EC 3.5.4.4) catalyzes the hydrolytic deamination reaction in which adenosine and deoxyadenosine is converted to inosine and deoxyinosine, respectively.

The active site of ADA contains a zinc atom, which participates directly in the deamination mechanism. The catalytic functions of the mammalian adenosine deaminases are carried out by side chains of some amino acids in the active region of the enzyme (Chang et al. 1991). The zinc

Correspondence to: Kadir Batcioglu, Inonu University, Faculty of Pharmacy, Department of Biochemistry, 44280 Malatya, Turkey E-mail: kadir.batcioglu@inonu.edu.tr ion is coordinated by His 15, His 17, His 214, and Asp 295. The possible conformational positions and interactions of amino acid residues have important roles for the activity of all enzymes and may change in the presence of a magnetic field (MF) (Berg et al. 2007).

MFs of various frequencies and strengths can be produced by high-voltage power lines, household appliances, industrial equipment and medical applications such as magnetic resonance imaging. Several studies have reported evidence for a relationship between the exposure to MFs and biological mechanisms such as cell proliferation, activity of many enzymes, membrane permeability, balance between intracellular and extracellular ion concentration, synthesis of DNA and RNA, immunological systems, production of free radicals, functions of the brain and cardiovascular systems, secretion of many hormones and activity of the central nervous system (Saunders and Jefferys 2007; Maslanyj et al. 2009)

The aim of this study has been an investigation of the effect of weak MFs on the activities of the purine-catabolyzing enzymes XOD and ADA in vitro. We used a MF intensity slightly above $5 \mathrm{mT}$, which is the maximum permissible under the guidelines from the International Non-ionizing Radiation Committee of the International Radiation Protection Association (IRPA/INIRC) for short term occupational 
whole body exposure (2 h per work day) (Jammet et al. 1990). To our knowledge, this is the first study of the effect of MF on XOD and ADA enzyme activities.

The applied MF was produced using a pair of Helmholtz coils. Two parallel coils were placed at a distance apart equal to the radius $r$. Subsequently, the magnitude of the MF at the centre of the coils, where the specimen was placed, was calculated using the equation below.

$$
\mathrm{H}=0.899 \mathrm{nI} / \mathrm{r}
$$

where $\mathrm{n}$ is the number of turns in each coil and I is the current. The MF system is shown in detail in Figure 1. In all the experiments, a $50 \mathrm{~Hz}$ uniform MF was generated and the magnitude of the field was kept constant at 5.8 $\mathrm{mT}$. The temperature of the system was monitored using a thermocouple which was connected next to the Helmholtz coil. No significant variation (less than $1^{\circ} \mathrm{C}$ ) was observed in the temperature of the system.

$3000 \mu \mathrm{l}$ of ADA solution with an activity of $200 \mathrm{U} / \mathrm{ml}$ was prepared. It was then divided into two equal volumes of $1500 \mu \mathrm{l}$. The first part of the enzyme solution was placed in the MF and the other part was kept five feet away from the MF. $50 \mu \mathrm{l}$ specimens were taken from each solution at the same time intervals and their activities were measured immediately. The activities of ADA solutions placed inside and outside the MF were measured for comparison purposes.
The same procedure was followed for the XOD solution with activity of $20 \mathrm{U} / \mathrm{ml}$.

Enzyme activities. The measurements were taken at 17 different time points between 0-250 minutes with differing intervals. All measurements of enzyme activity were taken twice and the averages used in the statistical analyses. Enzyme activities were expressed as units per $\mathrm{ml}(\mathrm{U} / \mathrm{ml})$.

The ADA activity was measured according to the method based on the deamination of adenosine to inosine (Giusti and Galanti 1984). In brief, ammonia liberated from adenosine reacts with phenol and hypochlorite to form the coloured compound indophenol, which is measured spectrophotometrically at $625 \mathrm{~nm}$. The absorbance of indophenol is proportional to the amount of ammonium. One unit of ADA activity is defined as the amount of enzyme required to liberate $1 \mu \mathrm{mol}$ of ammonium per minute from adenosine at $37^{\circ} \mathrm{C}$.

XOD activity was measured spectrophotometrically by the formation of uric acid from xanthine through the increase in absorbance at $293 \mathrm{~nm}$ (Prajda and Weber 1975). One unit of XOD activity was defined as $1 \mu \mathrm{mol}$ of uric acid formed per minute at $37^{\circ} \mathrm{C}$.

We employed simple linear regression of enzyme activity on the transformed time-differentials at each point of measurement for both the ADA and XOD enzymes. The response variables are the enzyme activities in presence of the MF (IM) and out of the MF (OF) of the MF, and the

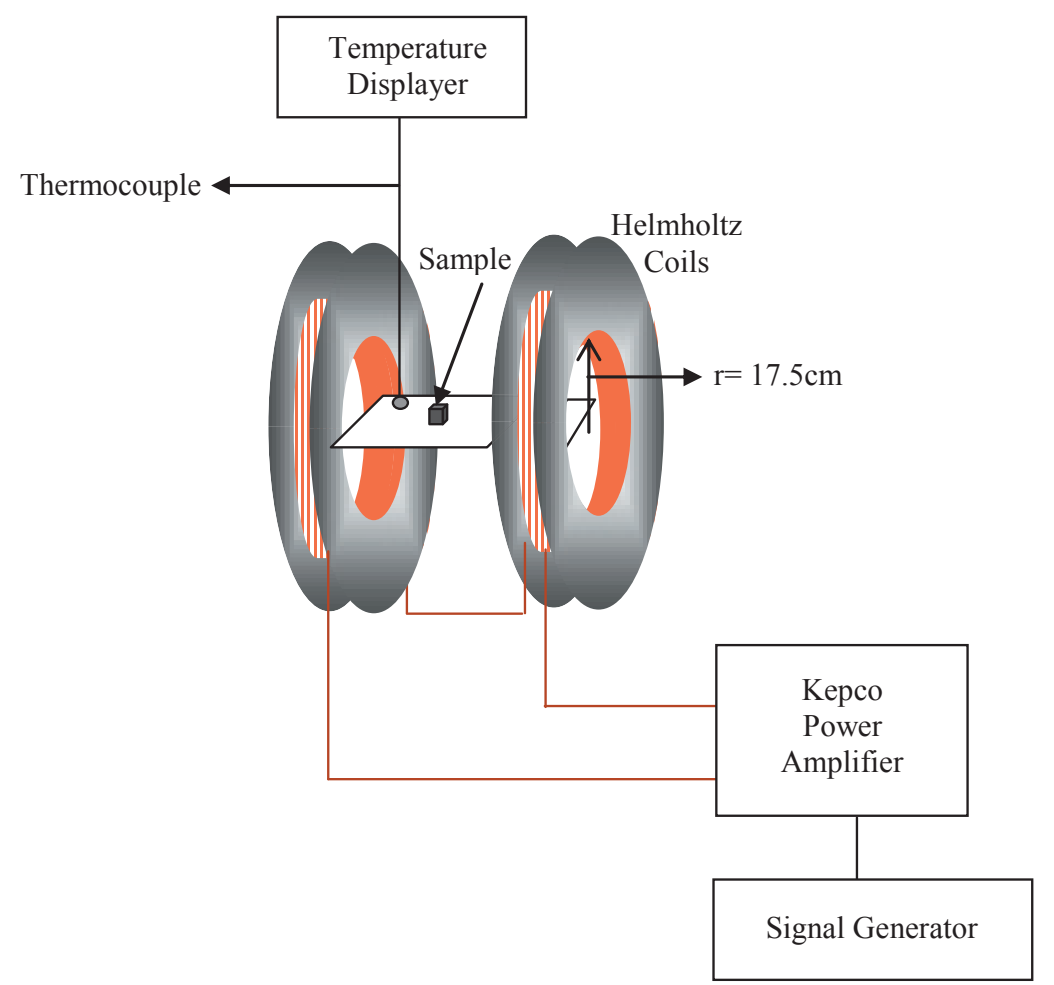

Figure 1. Magnetic field system. The power amplifier and signal generator were one foot away from the Helmholtz coils (the radius of the Helmholtz coil is $17.5 \mathrm{~cm}$ ). 
explanatory variable is time (T). We performed a simple linear regression analysis of IM $v s$. T and OM $v s$. T separately to compute the $95 \%$ confidence intervals of the regression coefficient relating to the times of measurements. In order to satisfy the linear regression assumptions, we used time differentials, delta(T), at each point rather than absolute time. In longitudinal studies, errors are inherently correlated, and to address the consequent violation of the assumption of independent errors, it is necessary to take the correlation between successive measurements into account. Regression diagnostics revealed that the constant variance assumption is not met. We handled this complication by using a square root transformation of delta(T). The final version of the explanatory variable was named sqrt(delta $(\mathrm{T}))$. Under this parameterization, the fundamental linear regression assumptions (i.e. errors are normally distributed and independent of each other, a conditional variance of the response variable given the predictor variable is constant) were satisfied, as demonstrated by standard residual plots versus orders, normal probability plots of residuals, among others. Summary statistics from the four separate regression equations are given in Table 1.

For the ADA, when a MF is present (see the ADAIM column in Table 1), the transformed time differentials turned out to be significant, as evidenced by high absolute T-value, low $\mathrm{P}$-value, high F-value and high R-squared (see Table 1). The estimate point of the sqrt(delta(T)) coefficient is -10.7378 . For further inferential validity, we constructed the $95 \%$ confidence interval for the regression coefficient of the transformed time difference. At the 0.05 significance level, the 95\% two-sided confidence interval with 15 degrees of freedom, where the theoretical t-value equals 2.131 , is $(-10.7378-2.131 \times 0.7507 ;-10.7378+2.131 \times 0.7507)=$ $(-12.3375 ;-9.1381)$. When a MF is absent (see the OM column for ADA in Table 1), the coefficient of the explanatory time variable is also highly significant, and the $95 \%$ two-sided confidence interval is $(-3.7815-2.131 \times 0.2884$; $-3.7815+2.131 \times 0.2884)=(-4.3961 ;-3.1669)$. It is clear that these two confidence intervals do not overlap, which leads us to infer that changes in enzyme activity over time are significantly different in and out of the MF. However, this result should be interpreted with caution. The argument we present is valid if the effect of the transformed time differential on enzyme activity is statistically significant in both regression equations and the enzyme activity is a semimonotonic (either non-decreasing or non-increasing with time) function of time under each experimental condition. Both requirements are satisfied by our experimental data. Therefore, we can state that the MF causes a steeper decline in ADA enzyme activity in comparison to the situation where no MF is present.

For the XOD, we conducted an identical analysis (see Table 1), using the same parameterization and arguments. In this particular case, the enzyme activity follows opposite trends in and out of the MF, since the regression slopes have different signs. Overall significance and semi-monotonocity requirements (apparent from the graphs), as well as the linear regression assumptions, are not violated for XOD under both experimental conditions. For this reason, we can utilize the same confidence interval comparison procedure. The 95\% confidence intervals for the coefficients of sqrt(delta $(\mathrm{T}))$ are $(0.8712-2.131 \times 0.1359 ; 0.8712+2.131 \times 0.1359)=(0.5816$; $1.1608)$, and $(-0.1546-2.131 \times 0.0083 ;-0.1546-2.131 \times$ $0.0083)=(-0.1724 ;-0.1368)$ with and without the MF, respectively. Due to the non-overlapping confidence intervals we conclude that the trend differences in the enzyme activity, with measurement time and with respect to the presence of the MF, are statistically significant. Another important result is that the MF led to an increase in the XOD activity. Experimental data are shown in Figure 2 and 3.

Some researchers have investigated the bio-usefulness of weak MFs. According to the results of these studies, MF activates natural killer cells (De Seze et al. 1993), stimulates IL-4 synthesis (Salerno et al. 1999), reduces the severity of pain (Colbert et al. 2008), and when antitumour drugs were used in combination with a weak MF the tumorigenesis was suppressed (Omote et al. 1990). In addition, thermal radiation has been known to play a very important role for a very

Table 1. Regression coefficients and other key statistical quantities of ADA and XOD enzymes in presence (IM) and absence $(\mathrm{OM})$ of the magnetic field

\begin{tabular}{lcccc}
\hline & \multicolumn{2}{c}{ ADA } & \multicolumn{2}{c}{ XOD } \\
& IM & OM & IM & OM \\
\hline Sqrt(delta(T)) & -10.7378 & -3.7815 & 0.8712 & -0.1546 \\
SE & 0.7507 & 0.2884 & 0.1359 & 0.0083 \\
T-value & -14.30 & -13.11 & 6.41 & -18.52 \\
P-value & 0.00 & 0.00 & 0.00 & 0.00 \\
F-value & 204.59 & 171.96 & 41.07 & 343.07 \\
$\mathrm{R}^{2}(\%)$ & 93.2 & 92.0 & 73.2 & 95.8 \\
\hline
\end{tabular}

ADA, adenosine deaminase; XOD, xanthine oxidase; SE, standard error (detailes see in text). 


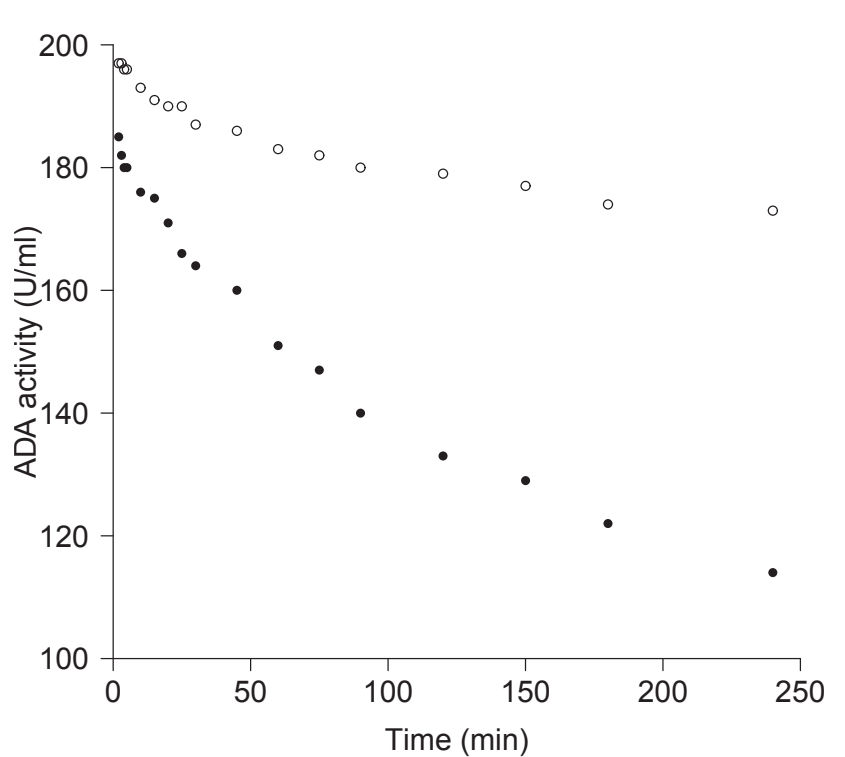

Figure 2. The activity of adenosine deaminase (ADA) in (O) and out $(\bullet)$ of the magnetic field.

long time in the treatment of various diseases. On the other hand, $\mathrm{Hu}$ et al. (2010) reported that the growth of injected melanoma cells was suppressed by a low intensity MF.

However, a many researchers seem to agree on the harmful effects of MFs. Some epidemiological studies suggest a possible link between long-term MF exposure and clinically recognized medical disorders, such as leukemia and many kinds of organ cancers, as well as cardiovascular and neurological diseases (Bethwaite et al. 2001; Hakansson et al. 2003; Labreche et al. 2003).

At this point, it is extremely difficult to say anything definite about the effects of MFs on living systems. Some findings concerning the harmful effects of strong and long-term MFs on biological systems have been reported by several researchers. However, others have reported that short-term exposure to low-frequency MF showed no destructive effect on cellular mechanisms. Moreover, it has been reported that a MF may be used as a facilitating factor during the treatment strategies of some diseases.

It has been known for a long time that the structural and catalytic properties of enzymes are affected by MFs. The three-dimensional structure of enzymes and substrates play a very important role during the catalytic activity of enzymes. It is possible that changes of the three-dimensional structure of enzymes brought about by a MF result in increased or decreased enzymatic activity. Another possible effect of a MF is modification of the oxidation/reduction potential of metal ions, which serve in the enzyme structure as cofactors.

The results of our previous in vitro study demonstrated that the MF increases the activity of superoxide dismutase (SOD), a reaction which needs $\mathrm{Cu}^{2+}$ as a cofactor for cata-

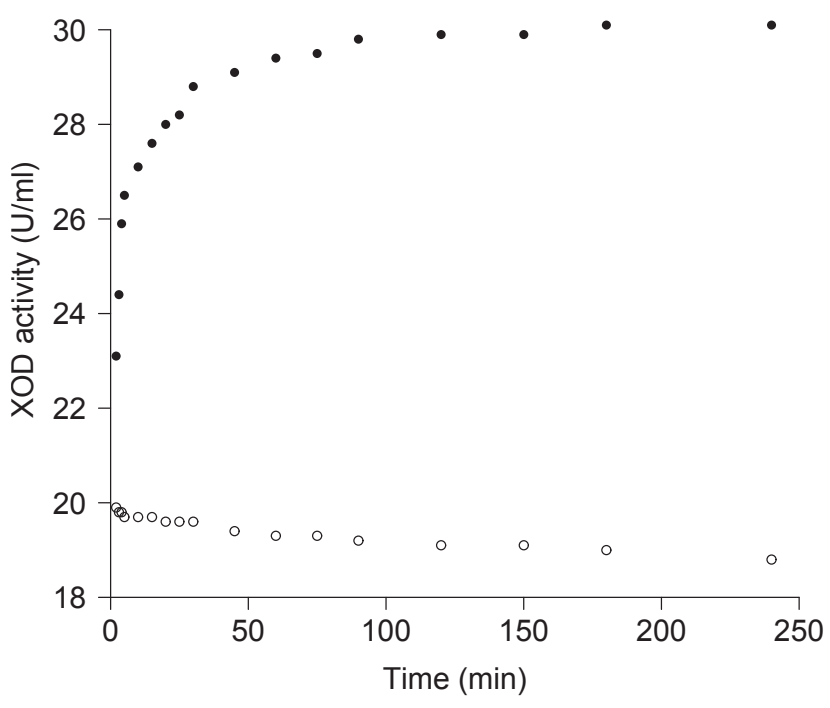

Figure 3. The activity of xanthine oxidase (XOD) in (O) and out $(\bullet)$ of the magnetic field.

lytic activity, whereas it decreases the activity of catalase (CAT) which uses $\mathrm{Fe}^{3+}$ as a cofactor (Batcioglu et al. 2002). In support of our findings, the inhibitory effect of a MF on thymidine kinase activity in mouse bone marrow cells has been reported by Feinendegen and Muhlensiepen (1987).

In conclusion, we found that the changes in ADA enzyme activity over time were significantly different in and out of MF. The MF caused a steeper decline in ADA enzyme activity when compared to the situation where no MF was present. However, the MF caused a significant increase in XOD enzyme activity. The major part of this increase occurred in the first few seconds but continued with smaller increases. There were no significant changes with time outside the MF for either enzyme. It is necessary to carry out further research to clarify the relationship between the MF and the activity of enzymes.

\section{References}

Batcioglu K., Ozturk I. C., Atalay S., Dogan D., Bayri N., Demirtas H. (2002): Investigation of time dependent magnetic field effects on superoxide dismutase and catalase activity: an in vitro study. J. Biol. Phys. Chem. 2, 108-112 http://dx.doi.org/10.4024/34020208.jbpc.02.03

Bethwaite P., Cook A., Kennedy J., Pearce N. (2001): Acute leukemia in electrical workers: a New Zealand case - control study. Cancer Causes Control 12, 683-689 http://dx.doi.org/10.1023/A:1011297803849

Berg J. M., Tymoczko J. L., Stryer L. (2007): Biochemistry. W. H. Freeman and Company, New York

Chang Z. Y., Nygaard P., Chinault A. C., Kellems R. E. (1991): Deduced amino acid sequence of Escherichia coli adenosine deaminase reveals evolutionarily conserved amino acid 
residues: implications for catalytic function. Biochemistry $\mathbf{3 0}$, 2273-2280

http://dx.doi.org/10.1021/bi00222a033

Colbert A. P., Markov M. S., Souder J. S. (2008): Static magnetic field therapy: dosimetry considerations. J. Altern. Complement. Med. 14, 577-582 http://dx.doi.org/10.1089/acm.2007.0827

De Seze R., Bouthet C., Tuffet S., Deschaux P. (1993): Effects of time-varying uniform magnetic fields on natural killer cell activity and antibody response in mice. Bioelectromagnetics 14, 405-412 http://dx.doi.org/10.1002/bem.2250140503

Doyle W. A., Burke J. F., Chovnick A., Dutton F. L., Whittle J. R., Bray R. C. (1996): Properties of xanthine dehydrogenase variants from rosy mutant strains of Drosophila melanogaster and their relevance to the enzyme's structure and mechanism. Eur. J. Biochem. 239, 782-795 http://dx.doi.org/10.1111/j.1432-1033.1996.0782u.x

Feinendegen L. E., Muhlensiepen H. (1987): In vivo enzyme control through a strong stationary magnetic field - the case of thymidine kinase in mouse bone marrow cells. Int. J. Radiat. Biol. 52, 469-479 http://dx.doi.org/10.1080/09553008714551931

Giusti G., Galanti B. (1984): Colorimetric method. In: Methods of Enzymatic Analysis. (Ed. H. U. Bergmeyer), pp. 315-323, Verlag Chemie, Weinheim

Hakansson N., Gustavsson P., Sastre A., Floderus B. (2003): Occupational exposure to extremely low frequency magnetic fields and mortality from cardiovascular disease. Am. J. Epidemiol. 158, 534-542 http://dx.doi.org/10.1093/aje/kwg197

Hu J. H., St-Pierre L. S., Buckner C. A., Lafrenie R. M., Persinger M. A. (2010): Growth of injected melanoma cells is suppressed by whole body exposure to specific spatial-temporal configurations of weak intensity magnetic fields. Int. J. Radiat. Biol. 86, 79-88 http://dx.doi.org/10.3109/09553000903419932

Jammet H. P., Bernhardt J. H., Bosnjakovic B. F. M., Czerski P., Grandolfo M., Harder D., Knave B., Marshall J., Repacholi M. H., Sliney D. H., Stolwijk J. A. J. (1990): Interim guidelines on limits of exposure to $50 / 60 \mathrm{~Hz}$ electric and magnetic fields. Health Phys. 58, 113-122
Labreche F., Goldberg M. S., Valois M. F., Nadon L., Richardson L., Lakhani R., Latreille B. (2003): Occupational exposures to extremely low frequency magnetic fields and postmenopausal breast cancer. Am. J. Ind. Med. 44, 643-652 http://dx.doi.org/10.1002/ajim.10264

Lacy F., Gough D. A., Schmid-Schonbein G. W. (1998): Role of xanthine oxidase in hydrogen peroxide production. Free Radic. Biol. Med. 25, 720-727 http://dx.doi.org/10.1016/S0891-5849(98)00154-3

Maslanyj M., Simpson J., Roman E., Schüz J. (2009): Power frequency magnetic fields and risk of childhood leukaemia: misclassification of exposure from the use of the distance from power line' exposure surrogate. Bioelectromagnetics 30, 183-188 http://dx.doi.org/10.1002/bem.20465

Omote Y., Hosokawa M., Komatsumoto M. (1990): Treatment of experimental tumors with a combination of a pulsing magnetic field and an antitumor drug. Jpn. J. Cancer Res. 81, 956-961 http://dx.doi.org/10.1111/j.1349-7006.1990.tb02673.x

Prajda N., Weber G. (1975): Malign transformation-linked imbalance: decreased XO activity in hepatomas. FEBS Lett. 59, 245-249 http://dx.doi.org/10.1016/0014-5793(75)80385-1

Salerno S., Lo Casto A., Caccamo N. (1999): Static magnetic fields generated by a $0,5 \mathrm{~T}$ MRI unit effects in vitro expression of activation markers and interleukin release in human peripheral blood mononuclear cells. Int. J. Radiat. Biol. 75, $457-463$ http://dx.doi.org/10.1080/095530099140384

Saunders R. D., Jefferys J. G. (2007): A neurobiological basis for ELF guidelines. Health Phys. 92, 596-603 http://dx.doi.org/10.1097/01.HP.0000257856.83294.3e

Takanami Y., Nakayama T. (2011): Evaluation of superoxide anion radicals generated from an aqueous extract of particulate phase cigarette smoke by electron spin resonance using 5,5dimethyl-1-pyrroline-N-oxide. Biosci. Biotechnol. Biochem. 75, 34-39

http://dx.doi.org/10.1271/bbb.100454

Received: January 27, 2011

Final version accepted: August 9, 2011 